\title{
Avaliação da eficiência da gestão dos serviços municipais de abastecimento de água e esgotamento sanitário utilizando Data Envelopment Analysis
}

\section{Evaluation of municipal service management efficiency of water supply and sanitation using Data Envelopment Analysis}

\author{
Dirceu Scaratti \\ Doutor em Engenharia de Produção pela Universidade Federal de Santa Catarina (UFSC) - Florianópolis (SC), Brasil. \\ Pesquisador e Professor na Universidade do Oeste de Santa Catarina (UNOESC) - Videira (SC), Brasil.
}

William Michelon

Estudante de Engenharia Sanitária e Ambiental na UNOESC - Videira (SC), Brasil.

Gidiane Scaratti

Mestranda em Engenharia Química na UFSC - Florianópolis (SC), Brasil.

\section{Resumo}

O presente estudo teve por objetivo avaliar a eficiência da gestão dos serviços municipais de abastecimento de água e esgotamento sanitário utilizandose da abordagem Data Envelopment Analysis. Foram avaliados 53 municípios prestadores de serviços de abastecimento de água potável e esgotamento sanitário, conforme definições da Lei 11.445/2007, com população residente entre 50 mil e 100 mil habitantes e constantes da amostra do Sistema Nacional de Informações sobre Saneamento. Utilizaram-se na avaliação 33 indicadores de desempenho cujos resultados foram transformados em medidas adimensionais de intervalo [0,1]. Da amostra total, nove serviços de abastecimento de água potável e três de esgotamento sanitário obtiveram avaliação com índice igual a 1 e classificação eficiente, sendo que os demais obtiveram avaliação inferior, ou seja, menores que 1 e classificação ineficiente. Na avaliação agregada da gestão do saneamento básico (abastecimento de água potável e esgotamento sanitário), somente um município obteve classificação eficiente.

Palavras-chave: eficiência; gestão; abastecimento de água; esgotamento sanitário; DEA.

\begin{abstract}
The present study aimed to evaluate the municipal management efficiency of water supply and sanitation using the approach of Data Envelopment Analysis. We evaluated 53 municipalities' service providers of potable water supply and sanitation, as definitions of Law 11.445/2007, with resident population between 50 thousand and 100 thousand inhabitants and from the sample of the National Information on Sanitation. Were used 33 indicators to evaluate the performance whose the results were transformed into dimensionless measures the interval $[0,1]$. Of the total sample, nine services potable water supply and three sanitation obtained evaluation with index equal 1 and efficient classification, and the others had a lower valuation, in other words, lower than 1 and inefficient ranking. In the aggregate evaluation of the management sanitation (water supply and sanitation), only one municipality had efficiency classification.
\end{abstract}

Keywords: efficiency; evaluation; water supply; sanitation; DEA. 


\section{Introdução}

O crescimento populacional elevou o consumo de água tratada e a respectiva geração de efluentes domésticos. Pesquisas relacionadas ao saneamento básico são publicadas constantemente, haja vista a estreita relação com as condições de saúde da população. As ações de saneamento podem ser consideradas preventivas para a saúde, quando garantem a qualidade da água de abastecimento e também da coleta, tratamento e disposição final adequada de dejetos humanos.

Após infindáveis discussões e proposições, a promulgação da Lei $n^{\circ} 11.445 / 2007$, que estabelece as diretrizes nacionais para o saneamento básico e para a política federal de saneamento básico, trouxe um alento inicial, porém, em pouco tempo percebeu-se que os desafios, nela propostos pelos princípios fundamentais de: (i) universalização, (ii) integralidade, (iii) disponibilidade, (iv) eficiência e sustentabilidade econômica, (v) segurança, qualidade e regularidade e (vi) integração das infraestruturas e serviços com a gestão eficiente dos recursos hídricos, exigirá grande esforço conjunto entre as esferas de governo e os poderes públicos para a sua implementação bem como para garantir recursos a serem destinados à ampliação do atendimento à população. Isto possibilitará evoluir positivamente em busca da universalização e da integralidade dos respectivos serviços associados ao saneamento básico.

Ademais, entre as principais funções das organizações de saneamento básico estão as de garantir a eficiência dos serviços demandados pela sociedade e de atender os requisitos legais exigidos pela legislação pertinente. Porém, há falhas e desperdícios no que se refere à eficiência nos serviços prestados, como, por exemplo, as perdas de água na distribuição de 38,8\% e de faturamento de 36,7\%, estimadas pelo Sistema Nacional de Informação sobre Saneamento - SNIS (BRASIL, 2012), além do alto consumo de energia elétrica do setor, cujos custos perdem apenas para a folha de pagamento na composição das despesas respectivas à gestão e operacionalização dos serviços públicos municipais de saneamento básico.

No Brasil, a gestão deste tipo de serviço se apresenta com grande diversidade de modelos, cada um com características específicas (LOUREIRO, 2009). A gestão, segundo a Lei no $11.445 / 2007$, delimita as atividades em abastecimento de água potável, esgotamento sanitário, limpeza urbana e manejo de resíduos sólidos, e drenagem e manejo das águas pluviais urbanas. O Decreto 7.217/2010, que regulamenta a referida Lei, estabelece como funções: planejamento, regulação, controle social, prestação dos serviços e aspectos econômicos e financeiros para os serviços públicos municipais de saneamento básico.

Dessa forma, fica evidente a necessidade de avaliar as organizações de saneamento básico de acordo com a sua infraestrutura, gestão, eficiência e sustentabilidade econômico-financeira e socioambiental, além da regularidade e continuidade dos serviços prestados. No entanto, o modelo de gestão adotado pelos serviços de saneamento é influenciado diretamente pela forma de prestação dos serviços adotados no município. Por isso, a área de saneamento tem muitos desafios a serem vencidos e a sua gestão está diretamente ligada ao bem-estar e à qualidade de vida da população (LOUREIRO, 2009).

Associar modelos de avaliação, com vistas a proporcionar ferramental adequado de avaliação da eficiência da gestão dos serviços municipais de abastecimento de água e esgotamento sanitário que contemple as infraestruturas detalhadas na atual legislação aos correspondentes serviços de saneamento básico, constitui-se em um paradigma cuja suplantação contribuirá para a elevação dos baixos índices de cobertura no atendimento, principalmente em esgotamento sanitário e, avançar para a universalização e a integralidade dos serviços associados ao saneamento básico no Brasil. A Tabela 1 apresenta os índices de atendimento dos serviços de água (abastecimento de água potável) e esgoto (esgotamento sanitário) resultantes da pesquisa do SNIS (BRASIL, 2012).

Modelos de avaliação têm-se utilizado da abordagem Data Envelopment Analysis (DEA) para avaliar a alocação eficiente de recursos (produtividade e eficiência técnica) e a qualidade da gestão de serviços em diversas áreas, empregando múltiplos insumos para gerar múltiplos produtos. Entre as publicações internacionais destacam-se os serviços da saúde (KIRIGIA et al., 2008; MURRAY; LOPEZ; WIBULPOLPRASERT, 2004; PINILLOS \& ANTOÑANZAS, 2002; RETZLAFF-ROBERTS; CHANG; RUBIN, 2004), enquanto as nacionais relatam a utilização do DEA em estudos econômicos da educação e saúde (CESCONETTO; LAPA; CALVO, 2008; FARIA; JANNUZZI; SILVA, 2008; GONÇALVES et al., 2007; LOBO et al., 2010; SCARATTI \& CALVO, 2012).

Especificamente na área do saneamento básico foram encontradas três publicações estrangeiras (THANASSOULIS, 2000; TUPPER \& RESENDE, 2004; YU \& WEN, 2010) e uma brasileira (CARMO \& TÁVORA, 2003).

Tabela 1 - Índice de atendimento dos serviços de água e esgoto.

\begin{tabular}{|c|c|c|c|c|c|}
\hline \multirow{3}{*}{ Região } & \multicolumn{4}{|c|}{$\begin{array}{l}\text { Índice com atendimento com rede } \\
\qquad(\%)\end{array}$} & \multirow{3}{*}{$\begin{array}{c}\text { Índice de } \\
\text { tratamento dos } \\
\text { esgotos gerados } \\
(\%) \\
\text { Total }\end{array}$} \\
\hline & \multicolumn{2}{|c|}{ Água } & \multicolumn{2}{|c|}{$\begin{array}{l}\text { Coleta de } \\
\text { esgoto }\end{array}$} & \\
\hline & Total & Urbano & Total & Urbano & \\
\hline Norte & 57,5 & 71,8 & 8,1 & 10,0 & 22,4 \\
\hline Nordeste & 68,1 & 87,1 & 19,6 & 26,1 & 32,0 \\
\hline Sudeste & 91,3 & 96,6 & 71,8 & 76,9 & 40,8 \\
\hline Sul & 84,9 & 96,0 & 34,3 & 39,9 & 33,4 \\
\hline Centro-Oeste & 86,2 & 95,3 & 46,0 & 50,5 & 43,1 \\
\hline Brasil & 81,1 & 92,5 & 46,2 & 53,5 & 37,9 \\
\hline
\end{tabular}

Fonte: Diagnóstico dos Serviços de Água e Esgoto do SNIS (BRASIL, 2012). 


\section{Metodologia}

Para a avaliação da eficiência da gestão dos serviços municipais de saneamento básico foram definidas curvas de desempenho ótimo, denominadas fronteiras de eficiência para índices iguais a 1,0 e de ineficiência para índices menores que 1,0. Considerou-se eficiente a gestão observada nos serviços municipais de saneamento (SMS) representados por pontos iguais a 1,00 , e ineficiente quando representados por pontos menores que 1,0. Ademais, quanto mais afastado da fronteira de eficiência for a classificação de um SMS, mais ineficiente é a sua gestão no saneamento básico. Neste contexto, o algoritmo utilizado para avaliar um SMS ${ }^{\circ}$ contemplou vários indicadores de desempenho de modo simultâneo (IDj, $\mathrm{j}=1,2, \ldots, \mathrm{J})$ e respectivas medidas $(\mathrm{Mj}, \mathrm{j}=1,2, \ldots, \mathrm{J})$ transformadas em funções monótonas e crescentes num intervalo [0, 1].

Considerados os valores observados $0 \leq \mathrm{mj} \leq 1$ das medidas $\mathrm{Mj}$, $j=1,2, \ldots, J$, resultantes da função desempenho apropriada e que não existem ou não são conhecidos padrões ótimos mj* para as medidas $\mathrm{Mj}, \mathrm{j}=1,2, \ldots, \mathrm{J}$, a gestão de um SMS ${ }^{\circ}$ é avaliada relativamente às gestões de outros SMS similares SMS ${ }^{\mathrm{n}}, \mathrm{n}=1,2, \ldots, \mathrm{N}$, também avaliados sob o prisma das medidas $\mathrm{Mj}, \mathrm{j}=1,2, \ldots, \mathrm{J}$.

Há diversos modelos matemáticos que podem ser aplicados para verificar se a gestão de uma organização é eficiente ou não. Muitos deles empregam DEA e assumem que gestores podem tomar decisões que levam as medidas $\mathrm{Ml}, \ldots, \mathrm{M}_{\mathrm{k}}$ a assumirem os valores $\mathrm{ml}, \ldots, \mathrm{m}_{\mathrm{K}}$, conforme indicado na Equação 1, tais que:

$\mathrm{m}_{\mathrm{k}}=\sum_{\mathrm{n}=0}^{\mathrm{N}} \mathrm{Z}_{\mathrm{n}} \cdot \mathrm{m}_{\mathrm{k}}^{\mathrm{n}}, \mathrm{k}=1,2, \ldots, \mathrm{K}_{\text {sempre que }}$

$\sum_{n=0}^{N} Z_{n}=1$ e $Z_{n} \geq 0, \forall n, n=0,1,2, \ldots, N$
O problema de verificar a existência de algum $\mathrm{SMS}^{\mathrm{n}}$ melhor que um $\mathrm{SMS}^{\circ}$ pode ser resolvido verificando se existem números $\mathrm{zn} \geq 0$, $\mathrm{n}=0,1,2, \ldots, \mathrm{N}$, como na Equação 2, tais que:

$\sum_{\mathrm{n}=0}^{\mathrm{N}} \mathrm{Z}_{\mathrm{n}}=1 \quad \mathrm{~m}_{\mathrm{k}}=\sum_{\mathrm{n}=0}^{\mathrm{N}} \mathrm{Z}_{\mathrm{n}} \cdot \mathrm{m}_{\mathrm{k}}^{\mathrm{n}} \geq \mathrm{m}_{\mathrm{k}}^{0}, \forall k$

existe algum $\mathrm{k}$ tal que

$\mathrm{m}_{\mathrm{k}}=\sum_{\mathrm{n}=0}^{\mathrm{N}} \mathrm{Z}_{\mathrm{n}} \cdot \mathrm{m}_{\mathrm{k}}^{\mathrm{n}}>\mathrm{m}_{1}^{0}$

Por sua vez, para verificar se existem tais $z_{n}$, foram adotadas as Equações 3 e 4, de programação linear:

Achar: $s_{k} \geq 0, k=1,2, \ldots K$ e $z_{n} \geq 0, n=0,1,2, \ldots, N$, que maximize $S=\sum_{n=1}^{K} S_{k}$

Tais que $\sum_{\mathrm{n}=0}^{\mathrm{N}} \mathrm{Z}_{\mathrm{n}}=1 \sum_{\mathrm{n}=0}^{\mathrm{N}} \mathrm{Z}_{\mathrm{n}} \cdot \mathrm{m}_{\mathrm{k}}^{\mathrm{n}}-\mathrm{S}_{\mathrm{k}}=\mathrm{m}_{\mathrm{k}}^{0}$

$\mathrm{k}=1,2, \ldots, \mathrm{K}$

Assim, para algum índice positivo $\left(S^{*}>0\right)$, a gestão de um $S M S^{\circ}$ é ineficiente, visto que existe indicador com medida positiva para um $s^{*} \mathrm{k}>0$, demonstrando a possibilidade dos gestores das organizações de saneamento básico diminuírem a medida de ineficiência desse SMS em pelo menos um dos indicadores sem prejudicar o desempenho em outro(s) indicador(es). Por outro lado, quando o índice for $\mathrm{S}^{*}=0$, a gestão é considerada eficiente, visto que o produto $s^{*} k=0$ para todo $k$ indica que a gestão do respectivo município SMS é eficiente. O Quadro 1 ilustra resumidamente o modelo esquemático adotado para agregar as medidas de eficiência da gestão dos serviços municipais que atuam em abastecimento de água potável e esgotamento sanitário.

Quadro 1 - Modelo esquemático de avaliação da eficiência da gestão.

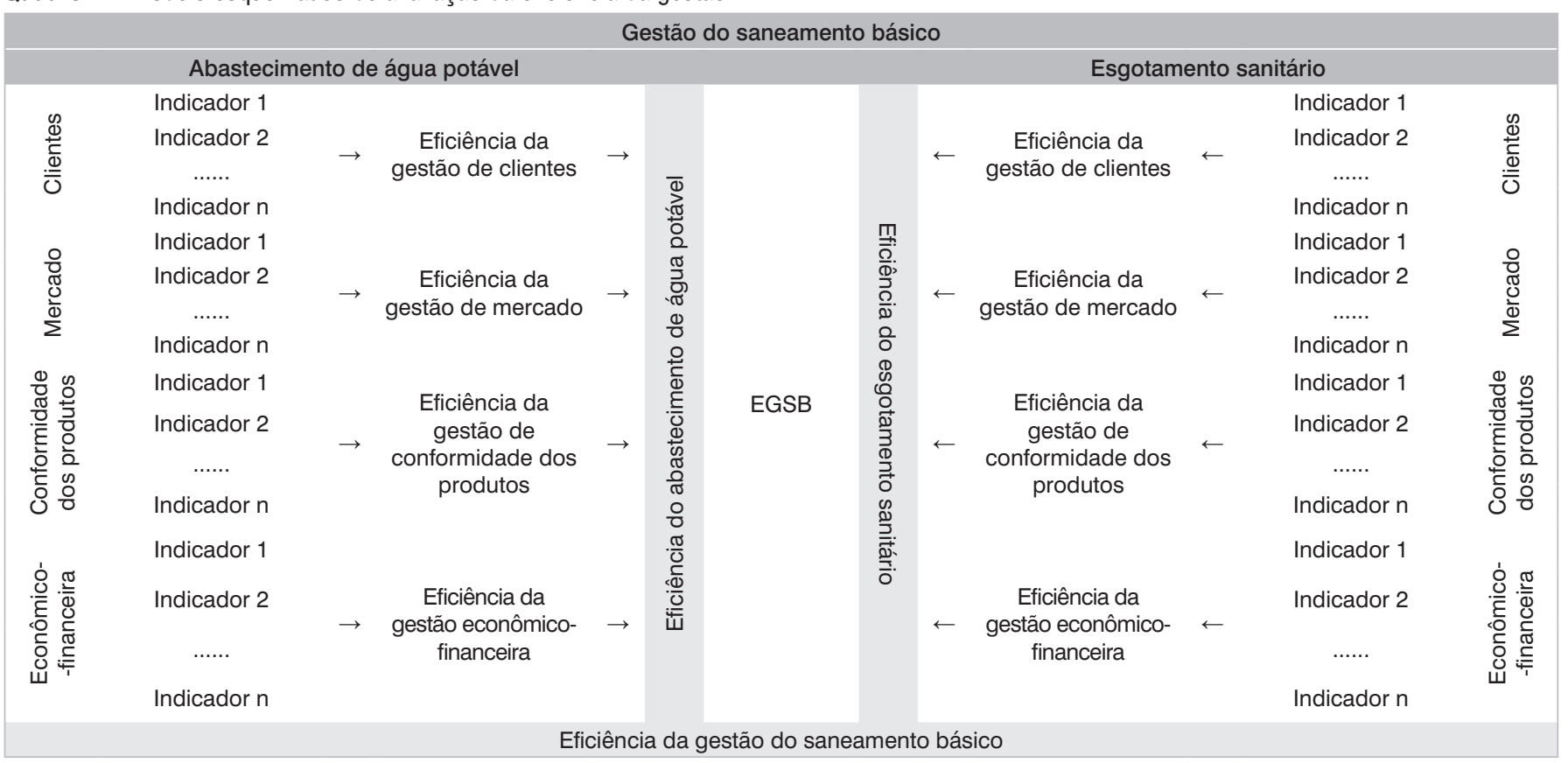


O modelo proposto avaliou a gestão do saneamento básico utilizando a abordagem DEA. A partir dele verificou-se a eficiência da gestão com o emprego de indicadores de desempenho de abastecimento de água potável e esgotamento sanitário relativos às perspectivas de clientes, mercado, conformidade dos produtos e a situação econômico-financeira, consolidados em um indicador sintético de gestão.

O algoritmo matemático foi empregado para verificar o desempenho de um serviço municipal de saneamento básico, comparando-o com o de outros serviços municipais. Portanto, a avaliação DEA, a partir deste algoritmo matemático, produziu medidas relativas que podem variar de um serviço municipal para outro. O algoritmo delineado para as agregações das medidas foi aplicado no software Lingo $^{\odot}$. A Tabela 2 apresenta os indicadores de desempenho adotados no modelo de avaliação.

Os dados necessários para empregar o modelo foram pesquisados nos bancos do Instituto Brasileiro de Geografia e Estatística (IBGE), do SNIS e do Programa de Modernização do Setor do Saneamento do Ministério das Cidades.
Os elementos do estudo foram municípios com população residente entre 50 mil e 100 mil habitantes, ano base 2010 (BRASIL, 2011a) e SNIS (BRASIL, 2011b), que possuíam infraestrutura instalada de abastecimento de água potável e esgotamento sanitário e integrantes da respectiva amostra do SNIS. Com isso, a aplicação restringiu-se a 53 municípios.

\section{Resultados}

$\mathrm{Na}$ aplicação, foram gerados relatórios para cada agregação de medidas em todos os indicadores de gestão do saneamento básico (abastecimento de água potável e esgotamento sanitário) a partir do algoritmo desenvolvido para a referida agregação. A Tabela 3 representa, para um município da amostra, as medidas de eficiência da gestão de clientes (EGC), de mercado (EGM), de conformidade dos produtos (EGCP) e econômico-financeira (EGEF), bem como a eficiência da gestão de abastecimento de água potável (EGAA), de esgotamento sanitário (EGES) e do saneamento básico (EGSB).

Tabela 2 - Indicadores de desempenho aplicados ao modelo de avaliação.

\begin{tabular}{|c|c|c|c|}
\hline \multirow[b]{2}{*}{ Perspectiva } & \multirow[b]{2}{*}{ Indicadores } & \multicolumn{2}{|c|}{ Dimensões de gestão } \\
\hline & & $\begin{array}{l}\text { Abastecimento de } \\
\text { água potável }\end{array}$ & $\begin{array}{l}\text { Esgotamento } \\
\text { sanitário }\end{array}$ \\
\hline \multirow{7}{*}{ Clientes } & Índice de macromedição & $\mathrm{x}$ & \\
\hline & Índice de hidrometração & $x$ & \\
\hline & Consumo médio de água por economia & $x$ & \\
\hline & Consumo de água faturado por economia & $x$ & \\
\hline & Consumo médio per capita de água & $x$ & \\
\hline & Índice de perdas na distribuição & $\mathrm{x}$ & \\
\hline & Índice de esgoto tratado referido à água consumida & & $x$ \\
\hline \multirow{6}{*}{ Mercado } & Índice de atendimento total de água & $x$ & \\
\hline & Índice de atendimento urbano de água & $x$ & \\
\hline & Índice de atendimento total de esgoto & & $x$ \\
\hline & Índice de atendimento urbano de esgoto & & $x$ \\
\hline & Índice de coleta de esgoto & & $\mathrm{x}$ \\
\hline & Índice de produtividade: economias ativas por pessoal total & $x$ & $x$ \\
\hline \multirow{6}{*}{ Conformidade dos produtos } & Índice de tratamento de esgoto coletado & & $x$ \\
\hline & Índice de perdas por ligação & $x$ & \\
\hline & Índice de fluoretação de água & $x$ & \\
\hline & Incidência das análises de cloro residual fora do padrão & $x$ & \\
\hline & Incidência das análises de turbidez fora do padrão & $x$ & \\
\hline & Incidência das análises de coliformes totais fora do padrão & $x$ & \\
\hline \multirow{10}{*}{ Econômico-financeira } & Despesa total com os serviços por $\mathrm{m}^{3}$ faturado & $\mathrm{x}$ & \\
\hline & Despesa de exploração por $\mathrm{m}^{3}$ faturado & $x$ & \\
\hline & Indicador de desempenho financeiro & $x$ & $\mathrm{x}$ \\
\hline & Índice de perdas no faturamento & $\mathrm{x}$ & \\
\hline & Margem da despesa com pessoal total & $\mathrm{x}$ & $x$ \\
\hline & Participação da despesa de pessoal versus despesa de exploração & $x$ & \\
\hline & Participação da despesa de energia elétrica versus despesa de exploração & $x$ & \\
\hline & Participação da receita operacional de água versus receita total & $x$ & \\
\hline & Participação da receita operacional de esgoto versus receita total & & $x$ \\
\hline & Dias de faturamento comprometidos com contas a receber & $x$ & $x$ \\
\hline
\end{tabular}


Tabela 3 - Medida da eficiência da gestão do saneamento básico por clientes, mercado, conformidade dos produtos e econômico-financeira em um município.

\begin{tabular}{|c|c|c|c|}
\hline \multirow{2}{*}{$\begin{array}{l}\text { Abastecimento de água } \\
\text { potável } \\
\text { Clientes }\end{array}$} & \multicolumn{2}{|c|}{$\begin{array}{l}\text { Medidas } \\
\text { agregadas }\end{array}$} & \multirow{2}{*}{$\begin{array}{c}\text { Esgotamento sanitário } \\
\text { Clientes }\end{array}$} \\
\hline & 0,63 & 0,02 & \\
\hline Mercado & 0,80 & 0,37 & Mercado \\
\hline $\begin{array}{l}\text { Conformidade dos } \\
\text { produtos }\end{array}$ & 0,71 & 1,00 & $\begin{array}{l}\text { Conformidade dos } \\
\text { produtos }\end{array}$ \\
\hline Econômico-financeira & 1,00 & 1,00 & Econômico-financeira \\
\hline $\begin{array}{l}\text { Abastecimento de água } \\
\text { potável }\end{array}$ & 0,81 & 0,69 & Esgotamento sanitário \\
\hline \multicolumn{4}{|c|}{ Eficiência da gestão do saneamento básico } \\
\hline \multicolumn{4}{|c|}{0,75} \\
\hline
\end{tabular}

O algoritmo matemático aplicado na abordagem DEA para agregar as medidas da perspectiva de mercado (com três indicadores de desempenho), município $n^{\circ} 1$ (M1) para a eficiência da gestão do abastecimento de água potável, foi o seguinte:

$\max =0 * z 1+0 * z 2+0 * z 3+, \ldots,+0 * z 53+1 * s 1+1 * s 2+1 * s 3$;

para $=z 1>=0 ; z 2>=0 ; z 3>=0, \ldots, z 53>=0 ; s 1>=0 ; s 2>=0 ; s 3>=0$;

Ind $1=1,00 * z 1+0,82 * z 2+0,21 * z 3+, \ldots,+1,00 * z 53-1 * s 1+0 * s 2+0 * s 3=0,97$;

Ind $2=1,00 * z 1+0,57 * z 2+1,00 * z 3+, \ldots,+1,00 * z 53+0 * s 1-1 * s 2+0 * s 3=0,95$;

Ind $3=0,28 * z 1+0,44 * z 2+0,09 * z 3+, \ldots,+0,53 * z 53+0 * s 1+0 * s 2-1 * s 3=0,68$;

Tal que $=1 * z 1+1 * z 2+1 * z 3+, \ldots,+1 * z 53=1$

END

Observa-se que os valores são apresentados para cada perspectiva e em cada dimensão de avaliação. Sendo assim, o valor que cada medida representada é o resultado da análise da respectiva eficiência da gestão. Contudo, os valores iguais a 1,00 indicam que o município encontra-se na fronteira daquela medida, ou seja, eficiente, e quanto menor o valor observado, mais distante do valor ideal para a determinada medida. A EGAA, bem como a EGES, indica o desempenho resultante da agregação dos valores de clientes, mercado, conformidade dos produtos e econômico-financeira. A agregação destas medidas resulta na EGSB.

O município representado na Tabela 3 tem gestão eficiente para a $\operatorname{EGEF}(1,00)$ no que se refere ao abastecimento de água potável. Todavia, o esgotamento sanitário apresentou eficiência na gestão em $\operatorname{EGCP}(1,00)$ e $\operatorname{EGEF}(1,00)$. Contudo, para a $\operatorname{EGC}(0,02)$ e $\operatorname{EGM}(0,37)$ da dimensão de esgotamento sanitário, e EGC $(0,63)$, EGM $(0,80)$ e EGCP $(0,71)$ para abastecimento de água, a gestão é ineficiente. Consequentemente, o desempenho resultante da agregação da $\operatorname{EGAA}(0,81)$ e EGES $(0,69)$ resultou em gestão ineficiente $(0,75)$, ou seja, este município pode melhorar 25 pontos percentuais, comparativamente aos demais 52 municípios da amostra.

Diante disso, em comparação aos outros 52 municípios avaliados, este tem gestão municipal de saneamento básico ineficiente. De modo semelhante, são geradas as medidas para cada um dos municípios avaliados. Os indicadores sintéticos para os serviços de abastecimento de água potável e esgotamento sanitário estão apresentados no Quadro 2. Nele, observa-se que nove municípios obtiveram gestão eficiente $(1,00)$ para o serviço de abastecimento de água potável (EGAA) e três para o de esgotamento sanitário (EGES).

A EGSB, que agrega os resultados dos serviços de abastecimento de água potável e esgotamento sanitário de forma concomitante, foi eficiente para apenas um município, embora seis deles tenham obtido ineficiência $\leq 0,10$.

O menor valor observado $(0,45)$ no Quadro 2 foi para a EGSB no município 20, enquanto a menor média dos indicadores sintéti$\cos$ (valores agregados) foi verificada para a EGES4 de esgotamento sanitário (0,229), e a maior média para a EGAAl de abastecimento de água potável $(0,904)$.

\section{Discussões}

Pesquisas iniciadas em 1957 (FARRELL) deram origem à abordagem DEA, e na década de 1970 já estavam consolidadas duas linhas de pesquisa que utilizam programação matemática para construir fronteiras de eficiência técnica: uma originária do trabalho de Charnes, Cooper e Rhodes (1978), que estuda medidas radiais e introduziu o termo DEA na literatura científica; e outra orientada pelo trabalho de Färe e Lovell (1978), que estuda medidas não radiais, sendo que estas permitem mudar o processo de produção, uma vez que possibilitam alterar as proporções dos produtos e dos insumos. A medida não radial mais utilizada é a Aditiva, apresentada por Charnes et al. (1985), adotada na construção do algoritmo.

O método de análise envoltória de dados utiliza-se de programação linear para construir uma superfície não paramétrica ou fronteira de dados, permitindo o cálculo das eficiências relativas a esta fronteira. O DEA propõe a determinação de uma função com os parâmetros a serem estimados e não associa aos modelos qualquer estrutura de probabilidade, porém, implicitamente gera uma função de produção via programação linear (GRIGOLIN, 2007).

De acordo com Carmo e Távora (2003), a metodologia DEA é composta por três estágios: (i) seleção das Decision Maker Units (DMUs), (ii) determinação dos inputs e outputs, e (iii) aplicação do modelo e análise dos resultados de acordo com o tipo de retorno de escala (constante ou variável) e o de abordagem (orientada a inputs ou outputs).

Um estudo desenvolvido no Reino Unido por Thanassoulis (2000), sobre o emprego do modelo DEA em agências regulatórias de água, demonstrou que o emprego da DEA serve como ótima ferramenta para medir o desempenho comparativo de organizações complexas, as quais funcionam melhor quanto maior for o número de DMUs e de variáveis avaliadas.

Por sua vez, Yu e Wen (2010) utilizaram a DEA para avaliar a evolução da sustentabilidade ambiental relativa a 46 cidades chinesas. Os resultados demonstraram que $24 \%$ das constantes na amostra são relativamente sustentáveis. 
Quadro 2 - Resultados agregados em indicadores sintéticos.

\begin{tabular}{|c|c|c|c|c|c|c|c|c|c|c|c|}
\hline \multirow[b]{2}{*}{ M } & \multicolumn{5}{|c|}{ Eficiência da gestão do abastecimento de água potável } & \multicolumn{5}{|c|}{ Eficiência da gestão do esgotamento sanitário } & \multirow[b]{2}{*}{ SIN_EGSB } \\
\hline & EGAA1 & EGAA2 & EGAA3 & EGAA4 & $\begin{array}{l}\text { SINT } \\
\text { EGAA }\end{array}$ & EGES1 & EGES2 & EGES3 & EGES4 & $\begin{array}{l}\text { SINT } \\
\text { EGES }\end{array}$ & \\
\hline 1 & 0,89 & 0,98 & 0,86 & 0,99 & 1,00 & 0,82 & 1,00 & 0,43 & 0,32 & 0,67 & 0,84 \\
\hline 2 & 1,00 & 0,80 & 0,69 & 0,65 & 0,81 & 0,67 & 0,80 & 0,30 & 0,11 & 0,50 & 0,66 \\
\hline 3 & 1,00 & 0,97 & 0,53 & 0,79 & 0,85 & 0,80 & 1,00 & 0,29 & 0,21 & 0,60 & 0,73 \\
\hline 4 & 0,93 & 0,55 & 0,51 & 0,79 & 0,72 & 0,77 & 1,00 & 0,44 & 0,26 & 0,65 & 0,69 \\
\hline 5 & 0,85 & 0,91 & 0,72 & 0,77 & 0,84 & 0,51 & 1,00 & 0,31 & 0,09 & 0,51 & 0,68 \\
\hline 6 & 0,95 & 0,93 & 0,74 & 0,78 & 0,87 & 0,68 & 1,00 & 0,35 & 0,17 & 0,58 & 0,73 \\
\hline 7 & 1,00 & 0,78 & 0,62 & 1,00 & 0,90 & 0,70 & 1,00 & 0,16 & 0,02 & 0,50 & 0,70 \\
\hline 8 & 1,00 & 0,32 & 0,63 & 0,33 & 0,59 & 0,72 & 1,00 & 0,47 & 0,26 & 0,64 & 0,62 \\
\hline 9 & 1,00 & 1,00 & 0,81 & 1,00 & 1,00 & 0,67 & 0,00 & 0,47 & 0,00 & 0,31 & 0,66 \\
\hline 10 & 1,00 & 0,99 & 0,80 & 1,00 & 1,00 & 0,72 & 1,00 & 0,56 & 0,33 & 0,68 & 0,84 \\
\hline 11 & 0,90 & 0,77 & 0,58 & 1,00 & 0,86 & 0,77 & 1,00 & 0,55 & 0,46 & 0,72 & 0,79 \\
\hline 12 & 1,00 & 0,98 & 1,00 & 0,93 & 1,00 & 0,81 & 0,17 & 0,93 & 0,12 & 0,54 & 0,77 \\
\hline 13 & 1,00 & 0,89 & 0,56 & 0,79 & 0,83 & 0,64 & 0,00 & 0,68 & 0,00 & 0,36 & 0,60 \\
\hline 14 & 0,50 & 0,85 & 0,89 & 1,00 & 0,87 & 0,09 & 0,00 & 1,00 & 0,00 & 0,30 & 0,59 \\
\hline 15 & 1,00 & 0,77 & 0,78 & 0,38 & 0,76 & 0,82 & 0,00 & 0,90 & 0,00 & 0,46 & 0,61 \\
\hline 16 & 1,00 & 1,00 & 0,81 & 1,00 & 1,00 & 1,00 & 0,00 & 0,70 & 0,00 & 0,52 & 0,76 \\
\hline 17 & 0,90 & 0,99 & 0,80 & 0,96 & 0,95 & 1,00 & 0,84 & 0,89 & 0,58 & 0,92 & 0,94 \\
\hline 18 & 1,00 & 0,95 & 0,76 & 0,80 & 0,90 & 0,64 & 0,01 & 0,88 & 0,01 & 0,41 & 0,66 \\
\hline 19 & 1,00 & 0,96 & 0,72 & 0,96 & 0,94 & 1,00 & 0,28 & 0,84 & 0,20 & 0,67 & 0,81 \\
\hline 20 & 0,36 & 0,66 & 0,88 & 0,19 & 0,55 & 0,49 & 0,00 & 0,80 & 0,00 & 0,35 & 0,45 \\
\hline 21 & 0,80 & 0,73 & 0,89 & 1,00 & 0,91 & 0,79 & 0,80 & 0,92 & 0,56 & 0,80 & 0,86 \\
\hline 22 & 1,00 & 1,00 & 0,80 & 0,78 & 0,94 & 0,64 & 1,00 & 0,19 & 0,02 & 0,49 & 0,72 \\
\hline 23 & 1,00 & 0,88 & 0,86 & 0,86 & 0,92 & 0,89 & 0,00 & 0,82 & 0,00 & 0,46 & 0,69 \\
\hline 24 & 1,00 & 0,82 & 0,79 & 1,00 & 0,95 & 0,77 & 0,00 & 1,00 & 0,00 & 0,47 & 0,71 \\
\hline 25 & 1,00 & 0,91 & 0,70 & 0,95 & 0,92 & 0,84 & 1,00 & 0,82 & 0,69 & 0,87 & 0,90 \\
\hline 26 & 0,95 & 0,96 & 1,00 & 0,80 & 0,95 & 0,92 & 0,02 & 0,94 & 0,02 & 0,52 & 0,74 \\
\hline 27 & 0,63 & 0,96 & 0,80 & 0,93 & 0,85 & 0,23 & 0,18 & 0,70 & 0,11 & 0,33 & 0,59 \\
\hline 28 & 0,85 & 0,95 & 0,78 & 0,80 & 0,87 & 0,93 & 0,00 & 0,84 & 0,00 & 0,49 & 0,68 \\
\hline 29 & 0,71 & 0,76 & 0,92 & 0,74 & 0,81 & 0,41 & 0,53 & 0,94 & 0,46 & 0,61 & 0,71 \\
\hline 30 & 0,88 & 0,91 & 0,84 & 0,71 & 0,86 & 0,85 & 0,33 & 0,92 & 0,24 & 0,61 & 0,74 \\
\hline 31 & 1,00 & 0,95 & 0,73 & 0,43 & 0,80 & 1,00 & 0,00 & 0,72 & 0,00 & 0,52 & 0,66 \\
\hline 32 & 1,00 & 0,84 & 0,83 & 0,82 & 0,90 & 0,82 & 0,00 & 0,92 & 0,00 & 0,46 & 0,68 \\
\hline 33 & 0,77 & 0,74 & 0,78 & 0,79 & 0,79 & 0,79 & 0,00 & 0,89 & 0,00 & 0,45 & 0,62 \\
\hline 34 & 0,87 & 0,97 & 0,87 & 0,92 & 0,93 & 0,89 & 1,00 & 1,00 & 1,00 & 1,00 & 0,97 \\
\hline 35 & 0,73 & 0,88 & 0,71 & 0,72 & 0,78 & 0,72 & 1,00 & 0,87 & 0,88 & 0,90 & 0,84 \\
\hline 36 & 0,87 & 0,84 & 0,81 & 0,87 & 0,87 & 0,88 & 0,00 & 0,91 & 0,00 & 0,48 & 0,68 \\
\hline 37 & 0,81 & 0,91 & 0,90 & 0,86 & 0,89 & 0,85 & 0,00 & 1,00 & 0,00 & 0,49 & 0,69 \\
\hline 38 & 0,74 & 0,94 & 0,76 & 0,93 & 0,87 & 1,00 & 1,00 & 0,85 & 0,74 & 1,00 & 0,94 \\
\hline 39 & 0,80 & 0,89 & 0,86 & 0,88 & 0,88 & 0,95 & 0,05 & 0,94 & 0,04 & 0,56 & 0,72 \\
\hline 40 & 0,88 & 0,95 & 0,86 & 1,00 & 1,00 & 1,00 & 1,00 & 0,95 & 0,69 & 1,00 & 1,00 \\
\hline 41 & 1,00 & 0,98 & 0,73 & 0,92 & 0,93 & 0,90 & 0,08 & 0,86 & 0,06 & 0,51 & 0,72 \\
\hline 42 & 1,00 & 0,98 & 0,76 & 0,71 & 0,89 & 0,68 & 0,00 & 0,85 & 0,00 & 0,41 & 0,65 \\
\hline 43 & 1,00 & 0,86 & 0,87 & 0,68 & 0,88 & 0,69 & 0,14 & 0,95 & 0,10 & 0,50 & 0,69 \\
\hline 44 & 1,00 & 0,92 & 0,80 & 0,68 & 0,87 & 0,75 & 0,00 & 0,93 & 0,00 & 0,45 & 0,66 \\
\hline 45 & 0,67 & 0,91 & 0,70 & 0,91 & 0,82 & 0,57 & 0,93 & 0,75 & 0,77 & 0,78 & 0,80 \\
\hline 46 & 1,00 & 1,00 & 0,80 & 1,00 & 1,00 & 0,67 & 1,00 & 0,17 & 0,19 & 0,54 & 0,77 \\
\hline 47 & 1,00 & 0,90 & 0,30 & 1,00 & 0,85 & 0,09 & 1,00 & 0,14 & 0,01 & 0,34 & 0,60 \\
\hline 48 & 1,00 & 0,95 & 0,83 & 1,00 & 1,00 & 0,79 & 1,00 & 0,29 & 0,14 & 0,58 & 0,79 \\
\hline 49 & 0,76 & 0,46 & 0,69 & 0,68 & 0,67 & 0,71 & 1,00 & 0,59 & 0,96 & 0,84 & 0,76 \\
\hline 50 & 1,00 & 0,71 & 0,80 & 0,63 & 0,81 & 1,00 & 1,00 & 0,37 & 0,02 & 0,69 & 0,75 \\
\hline 51 & 0,92 & 1,00 & 0,64 & 1,00 & 0,94 & 0,73 & 1,00 & 0,28 & 0,19 & 0,58 & 0,76 \\
\hline 52 & 1,00 & 0,98 & 0,78 & 1,00 & 0,99 & 1,00 & 1,00 & 0,84 & 0,65 & 0,96 & 0,98 \\
\hline 53 & 1,00 & 0,79 & 0,94 & 1,00 & 1,00 & 1,00 & 1,00 & 0,65 & 0,46 & 0,87 & 0,94 \\
\hline$x$ & 0,904 & 0,873 & 0,770 & 0,832 & 0,878 & 0,756 & 0,531 & 0,692 & 0,229 & 0,592 & 0,736 \\
\hline
\end{tabular}

M: municípios da amostra; EGAA: eficiência da gestão do abastecimento de água potável; EGAA1: eficiência da gestão econômico-financeira; EGAA2: eficiência da gestão da conformidade dos produtos; EGAA3: eficiência da gestão de mercado; EGAA4: eficiência da gestão de clientes; EGES: eficiência da gestão do esgotamento sanitário; EGES1: eficiência da gestão econômico-financeira; EGES2: eficiência da gestão da conformidade dos produtos; EGES3: eficiência da gestão de mercado; EGES4: eficiência da gestão de clientes; X: média dos valores. *Os valores "SINT" são resultantes da agregação dos indicadores de SINT EGAA: EGAA1, EGAA2, EGAA3, EGAA4 e da SINT EGES: EGES1, EGES2, EGES3 e EGES4. Por sua vez, o SINT EGSB é resultante da agregação das medidas de SINT EGAA e SINT EGES e corresponde à eficiência da gestão do saneamento básico. 
No Brasil, não há relatos na literatura de avaliação da eficiência da gestão dos serviços de abastecimento de água potável e esgotamento sanitário utilizando a DEA. Duas pesquisas semelhantes foram realizadas. Em uma delas, produzida por Carmo e Távora (2003), foi utilizada a abordagem DEA (retorno constante) para avaliar a eficiência técnica das empresas de saneamento no Brasil. Observou-se que 15 delas $(57,69 \%)$ foram eficientes. Quando verificadas sob o foco DEA-V (retornos variáveis de escala), 21 (80,77\%) foram consideradas eficientes.

O outro trabalho, conduzido por Tupper e Resende (2004), avaliou a eficiência de 20 companhias estaduais de água e esgoto durante o período 1996 a 2000. O estudo utilizou-se da DEA para medir a eficiência relativa a partir de três insumos (despesas de pessoal, custos operacionais e outros custos operacionais) e quatro produtos (água produzida, esgoto tratado, população atendida com água e população atendida com esgoto tratado).

A presente pesquisa apontou potencial de aplicação da abordagem DEA em processos avaliativos da gestão do saneamento básico, o que demonstrou também que deve haver maior importância na identificação das ineficiências em relação à qualidade dos serviços prestados, pois há poucos estudos aplicados à gestão de organizações do setor do saneamento básico. A utilização de um algoritmo matemático para construir um indicador sintético permitiu identificar as possibilidades de melhoria das ineficiências da gestão municipal do saneamento básico a partir de indicadores, perspectivas e dimensões de gestão.

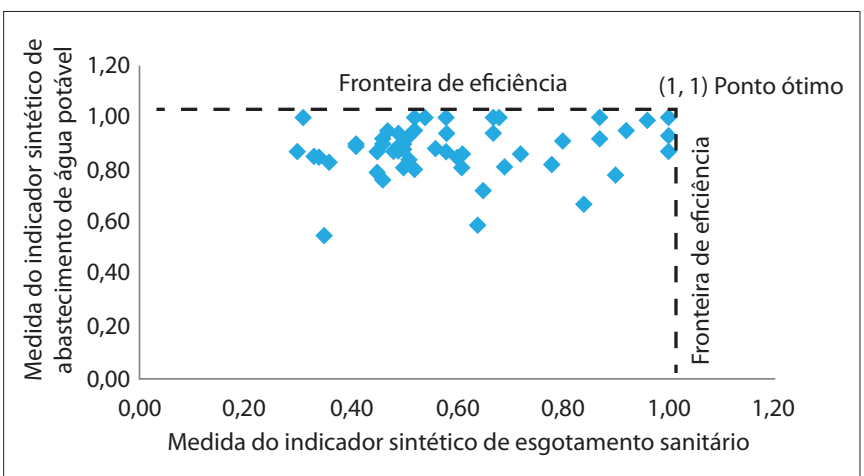

Figura 1 - Resultados sintéticos da eficiência do abastecimento de água potável e do esgotamento sanitário.

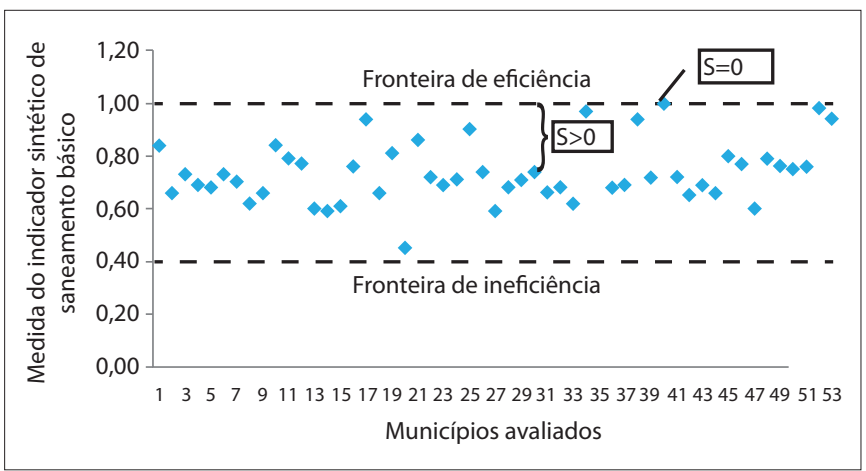

Figura 2 - Resultados sintéticos da eficiência da gestão do saneamento básico.
A visualização dos resultados finais da avaliação pode ser observada nas Figuras 1 e 2, a partir da geração das fronteiras de eficiência da gestão. A Figura 1 ilustra os indicadores sintéticos agregados das perspectivas: cliente, mercado, conformidade dos produtos e econômico-financeira para as dimensões abastecimento de água potável e esgotamento sanitário. Nela observa-se ainda o ponto ótimo $(1,1)$, conquistado por apenas um SMS, além de nove SMSs com EGAA e três com EGES.

Já na Figura 2 pode-se verificar os indicadores sintéticos entre os serviços de abastecimento de água potável e esgotamento sanitário por município. Apenas um município tem EGSB eficiente, com $\mathrm{S}=0$. Os demais 52 municípios têm valores $\mathrm{S}>0$, o que indica as suas ineficiências.

Embora os resultados mencionem apenas 1 SMS no ponto ótimo $(1,1)$ de eficiência da gestão municipal de saneamento básico, cabe ressaltar que 13,2\% dos SMSs analisados apresentaram índices $\geq 90,0 \%$ no desempenho $(0,90)$. Portanto, deve-se dar mais atenção à gestão dos respectivos serviços desempenhados e/ou concedidos pelos municípios, haja vista a importância e as oportunidades de melhorias disponíveis atualmente no Brasil no que se refere ao saneamento básico.

\section{Conclusão}

O modelo de avaliação apresentado teve como base a análise envoltória de dados para avaliar a eficiência da gestão dos serviços municipais de abastecimento de água potável e esgotamento sanitário, e os resultados finais indicam que os municípios, na sua grande maioria, têm importantes ineficiências associadas à gestão econômico-financeira, na conformidade dos produtos e serviços prestados e no atendimento ao mercado e aos clientes. Parte destas ineficiências pode ser creditada ao descaso político-governamental e ao baixo investimento no setor de saneamento básico que perdurou nas últimas décadas.

A DEA utilizada permitiu determinar a eficiência dos serviços de organizações e pode ser adotada de diversas formas, já que há poucos estudos relacionados à sua aplicação na avaliação da eficiência da gestão do saneamento básico. Ademais, o estudo foi de fundamental importância para demonstrar a ineficiência da gestão dos serviços municipais de saneamento, principalmente porque eles, no Brasil, não têm concorrência direta, o que credita à ineficiência uma das principais ameaças para o desempenho das organizações do setor.

Considerando-se que esta pesquisa contemplou em sua avaliação o saneamento básico nas dimensões de abastecimento de água potável e esgotamento sanitário, o modelo proposto pode ter sua aplicação estendida aos demais municípios brasileiros, e também incluir os serviços de manejo de resíduos sólidos urbanos e/ou drenagem urbana. 


\section{Referências}

BRASIL. (2007) Lei $n^{\circ} 11.445$, de 5 de janeiro de 2007. Estabelece diretrizes nacionais para o saneamento básico; altera as Leis nos 6.766 , de 19 de dezembro de 1979, 8.036, de 11 de maio de 1990, 8.666, de 21 de junho de 1993, 8.937, de 13 de fevereiro de 1995; revoga a Lei $n^{\circ}$ 6.528, de 11 de maio de 1978; e dá outras providências. Diário Oficial da União, Brasília, DF.

BRASIL. (2010) Decreto $n^{\circ} 7.217$, de 21 de junho de 2010. Regulamenta a Lei $n^{\circ} 11.445$, de 5 de janeiro de 2007, que estabelece diretrizes nacionais para o saneamento básico, e dá outras providências. Diário Oficial da União. Brasília.

BRASIL. Instituto Brasileiro de Geografia e Estatística - IBGE. (2011a) Censo demográfico 2010: características da população e dos domicílios. Resultados do universo. Rio de Janeiro: IBGE.

BRASIL. Ministério das Cidades. Secretaria Nacional de Saneamento Ambiental. (2011b) Sistema Nacional de Informações sobre Saneamento: diagnóstico dos serviços de água e esgotos - 2009. Brasília: MCIDADES. SNSA, $616 \mathrm{p}$.

BRASIL. Ministério das Cidades. Secretaria Nacional de Saneamento Ambiental. (2012) Sistema Nacional de Informações sobre Saneamento: diagnóstico dos serviços de água e esgotos - 2010. Brasília: MCIDADES. SNSA, $448 \mathrm{p}$.

CARMO, C.M.; TÁVORA, J.L. (2003) Avaliação da eficiência técnica das empresas de saneamento brasileiras utilizando a metodologia DEA. In: Encontro Nacional de Economia, 31 Anais... Rio de Janeiro: ANPEC.

CESCONETTO, A.; LAPA, J.S.; CALVO, M.C.M. (2008) Avaliação da eficiência produtiva de hospitais do SUS de Santa Catarina, Brasil. Caderno Saúde Pública, v. 24, n. 10, p. 2407-2417.

CHARNES, A.; COOPER, W.W.; GOLANY, B.; SEIFORD, L. (1985) Foundations of data envelopment analysis for Pareto-Koopmans efficient empirical production functions. Journal of Econometrics, v. 30, p. $91-107$.

CHARNES, A.; COOPER, W.W.; RHODES, E. (1978) Measuring the efficiency of decision making units. European Journal of Operational Research, v. 2, n. 4, p. 429-444.

FÄRE, R.; LOVELL, C.A.K. (1978) Measuring the technical efficiency of production. Journal of Economic Theory, v. 19, p. 150-162.

FARIA, F.P.; JANNUZZI, P.M.; SILVA, S.J. (2008) Eficiência dos gastos municipais em saúde e educação: uma investigação através da análise envoltória no estado do Rio de Janeiro. Revista de Administração Pública, v. 42, n.1, p. 155-177.
FARRELL, M. J. (1957) The measurement of productive efficiency. Journal of the Royal Statistical Society. Series A (General), v. 120, n. 3, p. 253-290.

GONÇALVES, A.C.; NORONHA, C.P.; LINS, M.P.E.; ALMEIDA, R.M.V.R. (2007) Análise envoltória de dados na avaliação de hospitais públicos nas capitais brasileiras. Revista Saúde Pública, v. 41, n. 3, p. 427-435.

GRIGOLIN, R. (2007) Setor de água e saneamento no Brasil: regulamentação e eficiência. Dissertação (Mestrado em Mercados Regulados), Fundação Getúlio Vargas, São Paulo, 64 p.

KIRIGIA, J.M.; EMROUZNEJAD, A.; CASSOMA, B.; ASBU, E.Z.; BARRY, S. (2008) A performance assessment method for hospitals: the case of municipal hospitals in Angola. Journal of Medical Systems, v. 32, n. 6, p. 509-519.

LOBO, M.S.C.; LINS, M.P.E.; SILVA, A.C.M.; FISZMAN, R. (2010) Avaliação de desempenho e integração docente-assistencial nos hospitais universitários. Revista de Saúde Pública, v. 44, n. 4, p. 581-590.

LOUREIRO, A.L. (2009) Gestão dos serviços de abastecimento de água e esgotamento sanitário no estado da Bahia: análise de diferentes modelos. Dissertação (Mestrado em Engenharia Ambiental Urbana), Universidade Federal da Bahia, Salvador, 187 p.

MURRAY, C.J.L.; LOPEZ, A.D.; WIBULPOLPRASERT, S. (2004) Monitoring global health: time for new solutions. British Medical Journal, v. 329, n. 7474, p. 1096-1100.

PINILLOS, M.; ANTOÑANZAS, F. (2002) La atención primária de salud: descentralización y eficiencia. Gaceta Sanitaria, v. 16, n. 5, p. 401-407.

RETZLAFF-ROBERTS, D.; CHANG, C.F.; RUBIN, R.M. (2004) Technical efficiency in the use of health care resources: a comparison of OECD countries. Health Policy, v. 69, n. 1, p. 55-72.

SCARATTI, D.; CALVO, M.C.M. (2012) Indicador sintético para avaliar a qualidade da gestão municipal da atenção básica à saúde. Revista de Saúde Pública, v. 46, n. 3, p. 446-455.

THANASSOULIS, E. (2000) The use of data envelopment analysis in the regulation of UK water utilities: water distribution. European Journal of Operational Research, v. 126, n. 2, p. 436-453.

TUPPER, H.C.; RESENDE, M. (2004) Efficiency and regulatory issues in the Brazilian water and sewage sector: an empirical study. Utilities Policy, v. 12, n. 1 , p. 29-40.

YU, Y.; WEN, Z. (2010) Evaluating China's urban environmental sustainability with Data Envelopment Analysis. Ecological Economics, v. 69, n. 9, p. 1748-1755. 